\title{
Características e potencial tecnológico da carne da capivara
}

\author{
Characteristcs and technological potential of the capybara meat
}

\author{
Marcos Franke Pinto ${ }^{I}$ Elisa Helena Giglio Ponsano"I \\ Ana Paula da Silva Almeida' ${ }^{\text {III }}$ Riana Jordão Barrozo Heinemann ${ }^{\text {IV }}$ \\ Wilson Machado de Souza ${ }^{\text {II }}$
}

\section{RESUMO}

A carne de capivara tem conquistado espaço no mercado de carnes exóticas, justificando estudos que auxiliem no conhecimento de suas características e no aperfeiçoamento do seu processo de obtenção. Com esse objetivo, neste trabalho, os músculos Longissimus dorsi e Semimembranosus provenientes de animal macho, com idade entre 12 e 18 meses, foram submetidos a procedimentos analíticos visando a avaliar suas características e seu comportamento quanto aos aspectos tecnológicos. A carne apresentou um baixo teor de gordura, de 0,4\% e 0,3\% para os músculos L.dorsi e Semimembranosus, respectivamente. Foi observada uma correlação inversa $(P<0,001)$ entre o comprimento do sarcômero e a força de cisalhamento. O comprimento de sarcômero do $m$. L. dorsi, em comparação com o $\mathrm{m}$. Semimembranosus, foi menor

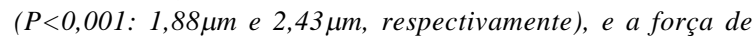
cisalhamento foi maior ( $P<0,001: 11,2 \mathrm{~kg}$ e $9,1 \mathrm{~kg}$, respectivamente), indicando ser a carne do pernil mais macia que o lombo. Além disso, a partir de aparas e vísceras, foram elaborados produtos cárneos, de acordo com formulaçõespadrão para hambúrguer, patê e mortadela. Esses produtos foram submetidos à análise sensorial, obtendo uma boa aceitabilidade.

Palavras-chave: capivara, carne, tecnologia, produtos cárneos.

\section{ABSTRACT}

The good rank capybara meat has reached within the exotic meat market justifies studies on its physical-chemical composition and also on the improvement of the production process. With that aim, Longissimus dorsi and

\begin{abstract}
Semimembranosus muscles of male animals ageing 12 to 18 months were evaluated about composition and technological properties. It was found a low fat content in capybara meat $0.4 \%$ and $0.3 \%$ for $\mathbf{L}$. dorsi and Semimembranosus muscles, respectively. A inverse correlation $(P<0.001)$ was found between sarcomere length and shear force. $L$. dorsi showed a shorter sarcomere lenght and a higher shear force $(P<0.001)$ than Semimembranosus muscle $(1.88 \mu \mathrm{m}$ and $2.43 \mu \mathrm{m} ; 11.2 \mathrm{~kg}$ and $9.1 \mathrm{~kg}$, respectively), what denotes that capybara ham meat is tenderer than loin meat. Moreover, trimmings and viscera were used in the elaboration of meat products as hamburger, pátê and mortadella. Sensory analysis of those products showed good acceptance.
\end{abstract}

Key words: capybara, meat, technology, meat products.

\section{INTRODUÇÃO}

Atualmente, nos grandes centros consumidores, observa-se a formação de um mercado de carnes exóticas. A carne de capivara tem se mostrado uma promissora alternativa para este mercado emergente, por suas características apreciadas. Hoje há uma tendência internacional de boicotar produtos de caça, o que tem aumentado bastante o interesse pela criação racional de animais silvestres para fins comerciais (PAIVA, 1992). A capivara pode ser criada nas áreas úmidas do cerrado, com tecnologia adaptada às condições do agricultor familiar, gerando a

\footnotetext{
IDepartamento de Apoio, Produção e Saúde Animal (DAPSA), Universidade Estadual Paulista (UNESP). R. Clóvis Pestana, 793, CP 341, 16050-680, Araçatuba, SP, Brasil. E-mail: mfpinto@fmva.unesp.br. Autor para correspondência.

IIDAPSA, UNESP, Araçatuba, SP, Brasil.

IIIPrograma de Pós-graduação em Ciência Animal, UNESP, Araçatuba, SP, Brasil.

${ }^{\text {Iv} P r o g r a m a ~ d e ~ T r e i n a m e n t o ~ T e ́ c n i c o ~ N i ́ v e l ~ I I I, ~ U N E S P, ~ A r a c ̧ a t u b a, ~ S P, ~ B r a s i l . ~}$
} 
possibilidade de renda, sem alterar a biodiversidade regional (AGROTEC, 2005). Além da carne, o interesse comercial pela capivara concentra-se na sua pele bastante elástica, resistente e suave, sendo ótima para a fabricação de luvas, bolsas e calçados (GONZALEZJIMENEZ, 1977; PAIVA, 1992).

A carne de capivara é considerada saudável pelo baixo teor de gordura (PAIVA, 1992) e pela composição dessa fração lipídica (FUKUSHIMA et al., 1997). As partes nobres, como pernil e lombo, já são comercializadas regularmente em alguns pontos específicos de venda, principalmente nas grandes cidades, porém apresentam preço elevado.

Assim, este estudo teve o objetivo de determinar as características físico-químicas da carne de capivara e avaliar o potencial tecnológico de produtos derivados, elaborados com a utilização de vísceras e aparas de carne. Com isso, busca-se gerar informações que auxiliem no conhecimento necessário para viabilizar o aumento do consumo da carne de capivara e agregar valor aos subprodutos da carcaça, aumentando a rentabilidade do processo.

\section{MATERIAL E MÉTODOS}

Obtenção e preparo das amostras

As carcaças e vísceras utilizadas foram provenientes de animais machos, com idade entre $12 \mathrm{e}$ 18 meses, abatidos no Frigorífico Pró-Fauna, especializado em abate de animais silvestres, localizado em Iguape-SP, de acordo com as normas e procedimentos oficiais, após prévio período de descanso em jejum e dieta hídrica, seguido de insensibilização, garantindo dessa forma a observância dos preceitos de abate humanitário (BRASIL, 1997).

Após o abate, as carcaças e vísceras foram imediatamente levadas à refrigeração por 48 horas. Em seguida, em ambiente refrigerado $\left(<10^{\circ} \mathrm{C}\right)$, foram obtidos cortes industriais, como pernil e lombo, sendo separadas as aparas de carne e gordura provenientes desse processo. Foram retiradas amostras dos músculos Longissimus dorsi e Semimembranosus. Essas amostras, juntamente com as aparas e as vísceras (fígado e coração), foram congeladas em câmara de congelamento $\mathrm{a}-25^{\circ} \mathrm{C}$. As aparas e as vísceras foram utilizadas nas formulações dos derivados cárneos. As amostras dos músculos Longissimus dorsi e Semimembranosus foram utilizadas para avaliação das características da carne descritas a seguir.

Determinação da composição química básica Os músculos Longissimus dorsi (lombo) e Semimembranosus (coxão mole), as vísceras (coração e fígado) e os produtos cárneos elaborados foram submetidos à determinação da composição química básica (umidade, gordura, proteína e cinzas), de acordo com os métodos descritos pela AOAC (1980).

Determinação do pH final

O pH dos músculos L. dorsi e Semimembranosus foi determinado 48 horas após o abate com aparelho Mettler modelo MP-120BLE.

Determinação da textura

A textura da carne foi avaliada instrumentalmente nos músculos $\boldsymbol{L}$. dorsi e Semimembranosus pela determinação da força de cisalhamento em Texturômetro TAXT2, equipado com lâmina de Warner Bratzler. Para a análise, as amostras foram processadas em forno a $170^{\circ} \mathrm{C}$ até atingirem $70^{\circ} \mathrm{C}$ internamente, então foram deixadas à temperatura ambiente $\left(25^{\circ} \mathrm{C}\right)$ até atingirem o equilíbrio térmico. Em seguida, foram retiradas de cada peça 6 amostras cilíndricas, de porções intermediárias da amostra (evitando-se porções periféricas e o ponto central), com $1,27 \mathrm{~cm}$ de diâmetro, no sentido longitudinal em relação à orientação das fibras. Esses cilindros de carne foram submetidos à determinação da força máxima de cisalhamento (POSTE et al., 1993).

\section{Análise microscópica}

Foi avaliado o grau de contração dos músculos estudados, pela determinação do comprimento dos sarcômeros, de acordo com a técnica padronizada por HEINEMANN et al. (2002). Para isso, amostras de cerca de três gramas dos músculos estudados foram acrescidas de solução de $\mathrm{KCl}$ 0,6M na razão de 1:5 (p/v) e homogeneizadas em aparelho tipo Stomacher por três minutos. Em seguida, uma alíquota de $0,1 \mathrm{ml}$ do homogenato obtido foi colocada em lâmina e observada em microscópio óptico acoplado a um sistema de captura de imagens. O comprimento do sarcômero foi determinado em pelo menos 20 campos para cada amostra, e os resultados expressaram a média dessas observações.

Estudo da viabilidade tecnológica dos produtos As aparas de carne e as vísceras (coração e fígado) foram empregadas na fabricação de hambúrguer, patê de fígado e mortadela, elaborados com base nas formulações descritas a seguir.

Formulações

Hambúrguer: carne de capivara (50,00\%), gordura suína (25,00\%), proteína texturizada de soja (10,00\%), água gelada e gelo (9,76\%), eritorbato de

Ciência Rural, v.37, n.3, mai-jun, 2007. 
sódio $(0,05 \%)$ e condimentos $(5,19 \%)$. Forma de processamento: moer a carne e a gordura, numa temperatura entre $0 \mathrm{e}-2^{\circ} \mathrm{C}$, em disco de $5 \mathrm{~mm}$, incorporar os ingredientes, modelar os hambúrgueres e congelar. Patê de fígado: carne de capivara (8,22\%), gordura suína e de capivara (18,26\%), fígado de capivara (19,17\%), água (45,62\%), nitrito de sódio (0,02\%), tripolifosfatos de sódio (0,40\%), eritorbato de sódio $(0,05 \%)$, proteína isolada de soja $(2,30 \%)$, glicose (1,60\%), estabilizante Globalfood ${ }^{\circledR}(1,70 \%)$, condimentos (2,66\%). Forma de processamento: colocar no cutter o fígado, a carne de capivara e a gordura, previamente cozidos, e os demais ingredientes (exceto os relacionados abaixo) e iniciar o processamento. Acrescentar proteína de soja, água a $80^{\circ} \mathrm{C}$, estabilizante e antioxidante. Misturar até a temperatura da massa atingir $40-42^{\circ} \mathrm{C}$. Embutir em filme de poliamida, processar em tacho de cozimento até atingir $75^{\circ} \mathrm{C}$ no ponto interno, resfriar em água corrente e armazenar em câmara de resfriamento.

Mortadela: carne de capivara (30,80\%), coração (5,00\%), gordura suína $(13,20 \%)$, proteína isolada de soja $(2,00 \%)$, nitrito de sódio $(0,02 \%)$, tripolifosfato de sódio $(0,40 \%)$, eritorbato de sódio (0,05\%), gelo (40,00\%), fécula de mandioca $(5,00 \%)$, corante e condimentos (3,53\%). Forma de processamento: triturar a carne, acrescentar sal, gelo, gordura, os demais ingredientes e, por último, a fécula. Embutir em tripa de poliamida, processar termicamente em tacho de cozimento até atingir $75^{\circ} \mathrm{C}$ no ponto interno, resfriar em água corrente e armazenar sob refrigeração.

\section{Análise sensorial}

Os produtos obtidos foram submetidos a testes afetivos de aceitabilidade para classificação, utilizando-se uma escala hedônica verbal de sete pontos (IFT, 1981; STONE \& SIDEL, 1993). As amostras foram apresentadas de forma monádica (amostras únicas) em cada sessão. Foram utilizados, no mínimo, 50 provadores não-treinados, e os dados obtidos foram ranqueados, segundo a porcentagem de consumidores que opinou por cada ponto da escala para cada produto testado (IFT, 1981).

\section{Análise estatística}

Os dados obtidos foram submetidos à análise descritiva. Para verificar as possíveis diferenças entre as médias, foi realizada análise de variância (ZAR, 1984). Os resultados obtidos para os testes de aceitação foram submetidos à análise pelo GLM, segundo o programa SAS (1985). Para verificar a significância entre as médias dos tratamentos, determinada pelo Teste $\mathrm{F}$, foi aplicado o teste de Duncan (ZAR, 1984).

\section{RESULTADOS E DISCUSSÃO}

Na determinação da composição química dos músculos e das vísceras, foi descartada a gordura ostensiva. Os músculos L. dorsi e Semimembranosus apresentaram 0,4 e 0,3\% de gordura, respectivamente. Já o fígado apresentou $0,4 \%$ de gordura e o coração 0,4\% (Tabela 1). ODA et al. (2004) encontraram teores de gordura de $0,83 \%$ e $0,36 \%$ em amostras de lombo e pernil de capivara, respectivamente. JARDIM et al. (2003) verificaram um teor de lipídeos variando de $0,54 \%$ a 1,3\%, em amostras do músculo $\mathbf{L}$. dorsi de capivaras, e demonstraram que esses valores são influenciados pelo peso dos animais e são significativamente mais elevados nas fêmeas. A carne avaliada neste trabalho foi proveniente de animais machos jovens, com idades entre 12 e 18 meses, criados e abatidos comercialmente, o que explica os resultados observados, uma vez que a gordura é um tecido de deposição mais tardia (LANNA, 1997).

Na tabela 1, são apresentados os valores de pH dos músculos estudados. São considerados normais valores de pH final entre 5,3 e 5,7 (JUDGE et al., 1989), mas a carne pode ser considerada boa para o consumo até um pH de 6,2 (LANARA, 1981). O estresse antes do abate reduz as reservas de glicogênio muscular, limitando a queda do $\mathrm{pH}$ da carne. Geralmente esse fenômeno é associado a fatores ambientais, mas pode haver participação de fatores intrínsecos, como um elevado metabolismo basal (TERRA, 1998). Pelo fato de serem animais silvestres, as capivaras podem apresentar uma maior suscetibilidade ao estresse, determinando uma depleção das reservas de glicogênio muscular e uma menor produção e acúmulo de ácido lático após o abate (HOFMANN, 1988; JUDGE et al., 1989). Embora indesejável sob o ponto de vista sanitário, um pH elevado traz vantagens tecnológicas,

Tabela 1 - Composição centesimal básica e pH dos músculos e das vísceras.

\begin{tabular}{lccccc}
\hline Amostra & $\begin{array}{c}\text { Umidade } \\
(\%)\end{array}$ & $\begin{array}{c}\text { Proteína } \\
(\%)\end{array}$ & $\begin{array}{c}\text { Gordura } \\
(\%)\end{array}$ & $\begin{array}{c}\text { Cinzas } \\
(\%)\end{array}$ & $\begin{array}{c}\text { Valores } \\
\text { de } \mathrm{pH}\end{array}$ \\
\hline Longissimus dorsi & 75,7 & 22,8 & 0,4 & 1,1 & 5,94 \\
Semimembranosus & 76,5 & 22,1 & 0,3 & 1,1 & 6,11 \\
Fígado & 74,1 & 22,0 & 0,4 & 1,3 & - \\
Coração & 77,4 & 20,1 & 0,2 & 0,9 & - \\
\hline
\end{tabular}


aumentando a capacidade de retenção de água da carne e permitindo a elaboração de produtos mais nobres, como presunto cozido (HONIKEL, 1988; JUDGE, 1989; TERRA, 1998).

Quando os músculos vermelhos são expostos ao frio em estágios anteriores ao estabelecimento do rigor mortis, há um aumento da força de contração tecidual (diminuição do comprimento do sarcômero), com conseqüente prejuízo da maciez da carne, fenômeno conhecido como encurtamento pelo frio (PARRISH et al., 1973; MARSH 1977; LOCHNER et al., 1980; JUDGE et al., 1989), o qual pode ser minimizado em carcaças grandes e pesadas, com adequada gordura de cobertura, pois a velocidade de resfriamento será menor (LOCHNER et al., 1980; MARSHALL, 1994). A carcaça da capivara é pequena e leve, se comparada a uma carcaça bovina. Além disso, as carcaças utilizadas neste trabalho apresentavam quantidade reduzida de gordura de cobertura. Ainda assim, os valores observados de comprimento de sarcômero (Tabela 2) foram semelhantes àqueles relatados para carne bovina (CULLER et al., 1978). Além disso, como ocorre na carne bovina, pode-se observar uma correlação inversa altamente significativa entre comprimento do sarcômero e força de cisalhamento (Tabela 3). O músculo $\boldsymbol{L}$. dorsi apresentou menor comprimento de sarcômero e maior força de cisalhamento (Tabela 2), o que pode ser atribuído à sua maior exposição ao frio.

A composição dos produtos elaborados (Tabela 4) e seu comportamento tecnológico, observado durante a elaboração, foram similares aos produtos tradicionais, obtidos a partir de carne suína e bovina. A análise sensorial demonstrou uma boa aceitação de todos os produtos (Figura 1), sendo que o hambúrguer foi classificado no ponto mais alto da escala, correspondente ao conceito "gostei extremamente”, por $51,1 \%$ dos consumidores. A mortadela e o patê de fígado foram classificados entre os escores 1 (gostei extremamente) e 3 (gostei

Tabela 2 - Comprimento do sarcômero e força máxima de cisalhamento dos músculos estudados

\begin{tabular}{lcc}
\hline Músculo & $\begin{array}{c}\text { Comprimento do } \\
\text { sarcômero }(\mu \mathrm{m})\end{array}$ & $\begin{array}{c}\text { Força de } \\
\text { cisalhamento }(\mathrm{kg})\end{array}$ \\
\hline Longissimus dorsi & $1,88^{*}$ & $11,16^{*}$ \\
Semimembranosus & 2,43 & 9,14 \\
\hline
\end{tabular}

$* \mathrm{P}<0,001$
Tabela 3 - Coeficiente de correlação de Pearson entre o comprimento do sarcômero (CS) e a força de cisalhamento (FC) dos músculos estudados

\begin{tabular}{lll}
\hline & CS $(\mu \mathrm{m})$ & FC $(\mathrm{kg})$ \\
\hline CS $(\mu \mathrm{m})$ & 1,00 & $-0,88^{*}$ \\
FC $(\mathrm{kg})$ & $-0,88$ & 1,00 \\
\hline
\end{tabular}

$* \mathrm{P}<0,001$.

moderadamente) por $90,8 \%$ e $96,4 \%$ dos provadores, respectivamente. O hambúrguer foi classificado nessa faixa por $97,9 \%$ dos provadores, e os $2,1 \%$ restantes o classificaram com escore 4, correspondente ao conceito “indiferente”. A mortadela apresentou a maior rejeição, recebendo o escore 5 (desgostei moderadamente) de $9,2 \%$ dos provadores. Deve-se considerar a possibilidade dessa rejeição ter ocorrido pelo elevado teor de sal, uma vez que o produto apresentou 4,3\% de cinzas (Tabela 4). O patê de fígado também apresentou uma pequena rejeição, sendo classificado com escore 5 (desgostei moderadamente) por 1,8\% dos provadores. Nenhum dos produtos recebeu escores 6 ou 7 , correspondentes aos conceitos "desgostei muito" e “desgostei extremamente”, respectivamente.

\section{CONCLUSÃO}

A carne da capivara apresentou valores elevados de força de cisalhamento, influenciados pelo grau de encurtamento muscular, sugerindo que o problema pode ser reduzido por um adequado manejo tecnológico. A utilização de aparas e vísceras na formulação de derivados cárneos revelou-se uma alternativa viável para aumentar a lucratividade do processo, o que pode proporcionar a possibilidade de redução do preço dos cortes mais nobres, tornando seu consumo acessível a uma faixa maior de consumidores.

\section{AGRADECIMENTOS}

Os autores agradecem à Fundação de Amparo à Pesquisa do Estado de São Paulo (Fapesp), pelo apoio financeiro.

Tabela 4 - Composição centesimal básica dos produtos cárneos

\begin{tabular}{lcccc}
\hline Produto & $\begin{array}{c}\text { Umidade } \\
(\%)\end{array}$ & $\begin{array}{c}\text { Proteína } \\
\mathbf{( \% )}\end{array}$ & $\begin{array}{c}\text { Gordura } \\
\mathbf{( \% )}\end{array}$ & $\begin{array}{c}\text { Cinzas } \\
\mathbf{( \% )}\end{array}$ \\
\hline Mortadela & 66,1 & 8,1 & 13,3 & 4,3 \\
Patê & 62,6 & 9,8 & 14,9 & 3,9 \\
Hambúrguer & 58,7 & 14,4 & 20,4 & 2,9 \\
\hline
\end{tabular}




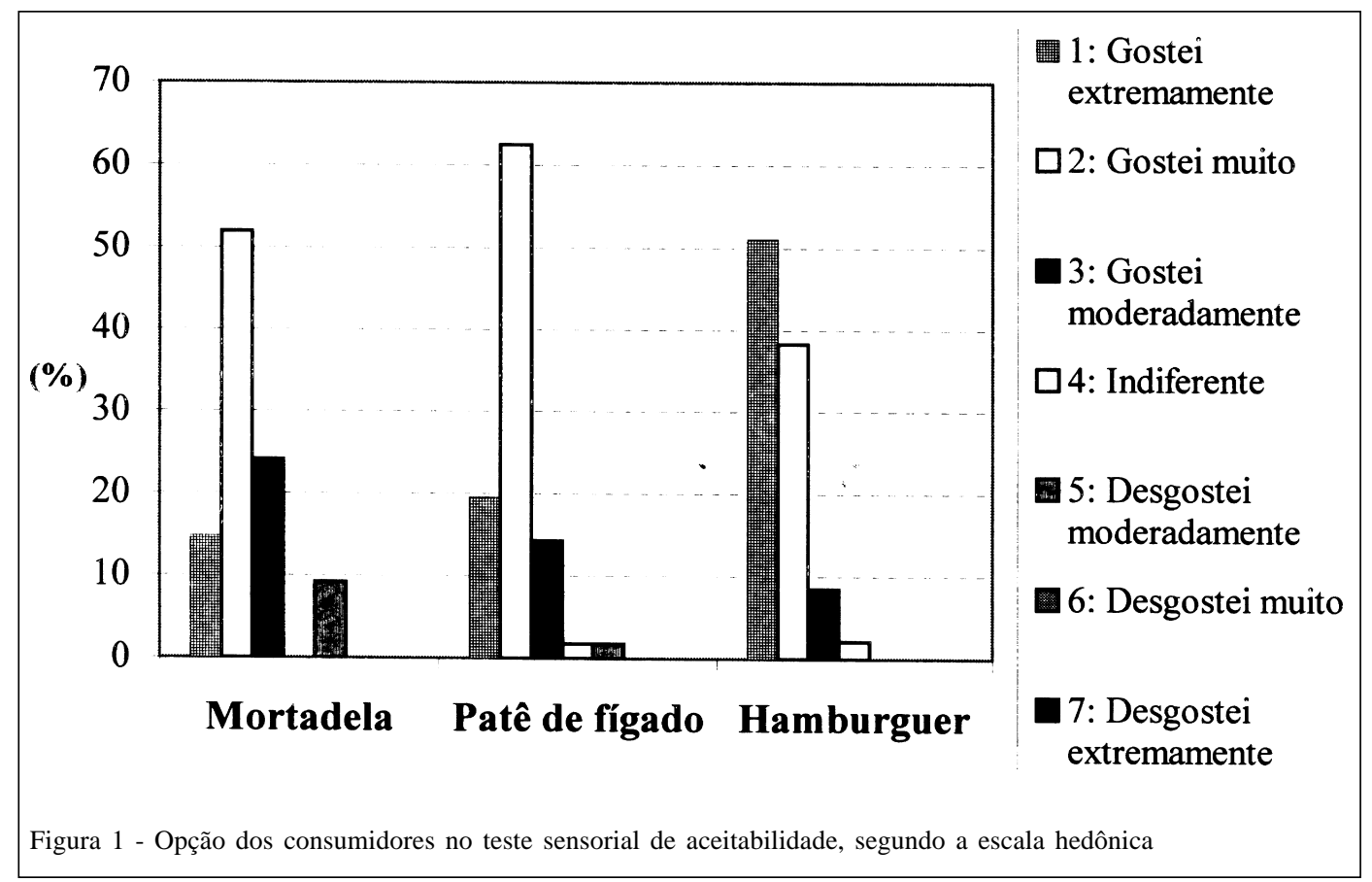

\section{REFERÊNCIAS}

AGROTEC. Região da vale do médio rio Araguaia: agricultura familiar: águas e produção sustentável. Portal de informações ambientais. Coalizão rios vivos. Acesso em 23 ago. 2005. Online. Disponível em: <http://www.riosvivos.org.br/ canal.php?canal=177>.

AOAC - ASSOCIATION OF OFFICIAL ANALYTICAL CHEMISTS. Official methods of analysis. 13.ed. Washington, 1980. 1015p.

BRASIL. Decreto no 2244, de 5 de junho de 1997. Estabelece regulamentação da inspeção industrial e sanitária de produtos de origem animal. Diário oficial da República Federativa do Brasil, poder Executivo, Brasília, DF, 4 de jun. 1997. Seção I, p.204.

CULLER, R.D. et al. Relationship of myofibril fragmentation index to certain chemical, physical and sensory characteristics of bovine longissimus muscle. Journal of Food Science, v.43, n.4, p.1177-1180, 1978.

FUKUSHIMA, M. et al. Comparative hypocholesterolemic effects of capybara (Hidrochoerus hidrochaeris dabbenei) oil, horse oil and sardine oil in cholesterol-fed rats. Lipids, v.32, n.4, p.391-395, 1997.

GONZALEZ-JIMENEZ, E. The capybara: an indigenous source of meat in tropical America. World Animal Review, n.21, p.24-30, 1977.

HEINEMANN, R.J.B. et al. Método simples para estimar encurtamento pelo frio em carne bovina. Ciência Rural, v.32, n.2, p.337-341, 2002.

HOFMANN, K. El pH: una característica de calidad de la carne. Fleischwirtschaft, v.1, p.13-18, 1988.
HONIKEL, K.O. Capacidad de fijación de agua de la carne. Fleischwirtschaft, v.1, p.3-12, 1988.

IFT - INSTITUTE OF FOOD TECHNOLOGISTS. Sensory evaluation guide for testing food and beverage products. Food Technology, v.35, n.11, p.50-59, 1981.

JARDIM, N.S. et al. Teor lipídico e perfil de ácidos graxos da carne de capivara (Hydrochaeris hydrochaeris). Ciência Agrotécnica, v.27, n.3, p.651-657, 2003.

JUDGE, M.D. et al. Principles of meat science. 2.ed. Iowa: Kendall Hunt, 1989. 351p.

LANARA. Métodos analíticos oficiais para controle de produtos de origem animal e seus ingredientes. Brasília: Ministério da Agricultura, 1981. 2v.

LANNA, D.P. Fatores condicionantes e predisponentes da puberdade e da idade de abate. In: SIMPÓSIO SOBRE PECUÁRIA DE CORTE, 4., 1996, Piracicaba. Anais... Piracicaba: FEALQ, 1997. p.79-97.

LOCHNER, J.V. et al. Early postmortem cooling rate and beef tenderness. Meat Science, v.4, n.3, p.227-241, 1980.

MARSH, B.B. Basis of tenderness in muscle foods. Journal of Food Science, v.42, n.2, p.295-297, 1977.

MARSHALL, D.M. Breed differences and genetic parameters for body composition traits in beef cattle. Journal of Animal Science, v.72, n.10, p.2745-2755, 1994.

ODA, S.H.I. et al. Composição centesimal e teor de colesterol dos cortes comerciais de capivara capivara (Hydrochaeris hydrochaeris). Ciência Agrotécnica, v.28, n.6, p.1344-1351, 2004.

Ciência Rural, v.37, n.3, mai-jun, 2007. 
PAIVA, R. Capivara: bicho novo no pasto. Globo Rural, n.80, p.42-47, 1992.

PARRISH, F.C. et al. Effect of postmortem conditions on certain chemical, morphological and organoleptic properties of bovine muscle. Journal of Food Science, v.38, p.690-695, 1973.

POSTE, L.M. et al. Correlations of sensory and instrumental meat tenderness value as affected by sampling techniques. Food Quality and Preference, v.4, n.4, p.207-214, 1993.
SAS Institute. SAS user's guide: statistics. 5.ed. Cary, 1985. 584p

STONE, H.; SIDEL, J.L. Sensory evaluation pratices. 2.ed. London: Academic, 1993. 338p.

TERRA, N.N. Apontamentos sobre tecnologia de carnes. São Leopoldo: Unisinos, 1998. 216p.

ZAR, J.H. Biostatistical analysis. 2.ed. Englewood Cliffs: Prentice Hall International, 1984. 718p. 